\title{
NORMALITY OF $\sigma$-PRODUCTS
}

\author{
KEIKO CHIBA \\ (Communicated by Franklin D. Tall) \\ Dedicated to Professor Akihiro Okuyama on his 60th birthday
}

\begin{abstract}
We prove that no $\sigma$-product of an uncountable family of nontrivial spaces is hereditarily normal or hereditarily $\omega_{1}$-paracompact. Also, if every finite subproduct of a $\sigma$-product is (hereditarily) countably paracompact and (hereditarily) normal, then the $\sigma$-product is (hereditarily) countably paracompact iff it is (hereditarily) normal.
\end{abstract}

\section{INTRODUCTION}

We later define $\sigma$-products which were introduced by Corson [3].

Throughout this paper we assume that $\kappa$ is an infinite cardinal, $\mathscr{S}=$ $\left\{X_{\alpha} \mid \alpha<\kappa\right\}$ where each $X_{\alpha}$ is a Hausdorff space having at least two points, and $\sigma$ is a $\sigma$-product of $\mathscr{S}$.

Kombarov proved:

(A) (Kombarov [4, Theorem 3]). If every finite subproduct of $\sigma$ is paracompact, then $\sigma$ is paracompact.

Further he essentially showed:

(B) If $\sigma$ is normal and $\lambda$ is an infinite cardinal and every finite subproduct of $\sigma$ is $\lambda$-paracompact, then $\sigma$ is $\lambda$-paracompact.

Here $\prod_{\alpha \in F} X_{\alpha}$ is said to be a finite subproduct of $\sigma$ if $F$ is a finite subset of $\kappa$.

We prove:

Theorem 1. If $\mathscr{S}$ is uncountable, $\sigma$ is not hereditarily normal.

Theorem 2. If $\mathscr{S}$ is uncountable, $\sigma$ is not hereditarily $\omega_{1}$-paracompact.

Theorem 3. If $\mathscr{S}$ is countable and every finite subproduct of $\sigma$ is countably paracompact and normal, then $\sigma$ is countably paracompact iff $\sigma$ is normal.

Theorem 4. If $\mathscr{S}$ is countable and every finite subproduct of $\sigma$ is hereditarily countably paracompact and hereditarily normal, then $\sigma$ is hereditarily countably paracompact iff $\sigma$ is hereditarily normal.

Received by the editors January 3, 1992 and, in revised form, March 13, 1992.

1991 Mathematics Subject Classification. Primary 54B05, 54B10; Secondary 54D15, 54D20.

Key words and phrases. $\sigma$-product, normal, countably paracompact, hereditarily normal, hereditarily countably paracompact. 


\section{Preliminaries}

By definition, " $\sigma$ is a $\sigma$-product of $\mathscr{S}$ " means there is a point $x^{*} \in X=$ $\prod_{\alpha<\kappa} X_{\alpha}$ (called the base point of $\sigma$ ) such that $\sigma$ is the subspace of $X$ consisting of $\left\{x \in X \mid\left\{\alpha \in \kappa \mid x(\alpha) \neq x^{*}(\alpha)\right\}\right.$ is finite $\}$.

We use standard set-theoretic notation including, for a set $B, B^{<\omega}=\{$ finite subset of $B$ \} and, for $n \in \omega, B^{n}=$ \{subset of $B$ of cardinality $\left.n\right\}$. If $B \subset A \subset X, \operatorname{cl} B$ means the closure of $B$ in $X$ while $\operatorname{cl}_{A} B$ means the closure of $B$ in $A$.

A space is called $\lambda$-paracompact [5] for some infinite cardinal $\lambda$ if every open cover of the space of cardinality $\leq \lambda$ has a locally finite open refinement. It is (countably) metacompact [2] if every (countable) open cover has a point finite open refinement.

For $a \in \kappa^{<\omega}$, let $Y_{a}=\prod_{\alpha \in a} X_{\alpha} \times \prod_{\alpha \in \kappa-a}\left\{x^{*}(\alpha)\right\}$. Let $p_{a}: \sigma \rightarrow Y_{a}$ be the projection map defined by

$$
p_{a}(x)(\alpha)= \begin{cases}x(\alpha) & \text { if } \alpha \in a, \\ x^{*}(\alpha) & \text { if } \alpha \in \kappa-a .\end{cases}
$$

For $a, b \in \kappa^{<\omega}$, with $a \subset b$, define $p_{a}^{b}: Y_{b} \rightarrow Y_{a}$ by

$$
p_{a}^{b}(x)(\alpha)= \begin{cases}x(\alpha) & \text { if } \alpha \in a, \\ x^{*}(\alpha) & \text { if } \alpha \in b-a .\end{cases}
$$

Obviously $p_{a}$ and $p_{a}^{b}$ are continuous open maps and $p_{a}^{b} \circ p_{b}=p_{a}$ when $a \subset b$.

\section{Proof of OUR THEOREMS}

Proof of Theorem 1. Let $\mathscr{D}=\left\{2_{\alpha} \mid \alpha<\omega_{1}\right\}$, where, for each $\alpha<\omega_{1}, 2_{\alpha}$ is a copy of the discrete space $\{0,1\}$ of two points. Let $d^{*} \in D=\prod_{\alpha<\omega_{1}} 2_{\alpha}$ be defined by $d^{*}(\alpha)=0$ for all $\alpha<\omega_{1}$. If $\sigma(\mathscr{D})$ is the $\sigma$-product of $\mathscr{D}$ with base point $d^{*}$, then, for all uncountable $\mathscr{S}$, there is a homeomorphic embedding of $\sigma(\mathscr{D})$ in $\sigma$ with $x^{*}$ as the image of $d^{*}$. Thus it suffices to prove:

Proposition 1. $\sigma(\mathscr{D})-\left\{d^{*}\right\}$ is not normal.

Proof. Let $B$ and $C$ be uncountable disjoint subsets of $\omega_{1}$. For $\alpha \in \omega_{1}$, let $f_{\alpha}$ be the point of $D$ defined by $f_{\alpha}(\alpha)=1$ but $f_{\alpha}(\beta)=0$ for all $\beta \neq \alpha$. Let $E=\left\{f_{\alpha} \mid \alpha \in B\right\}$ and $F=\left\{f_{\alpha} \mid \alpha \in C\right\}$. Since $d^{*}$ is the only limit point of $E$ or $F$ in $D, E$ and $F$ are closed and disjoint in $\sigma(\mathscr{D})-\left\{d^{*}\right\}$. Suppose $L$ is open in $\sigma(\mathscr{D})-\left\{d^{*}\right\}$ and $E \subset L$. We find a point of $F$ in the closure of $L$, thus proving that $\sigma(\mathscr{D})-\left\{d^{*}\right\}$ is not normal.

For each $\alpha \in B$ there is a finite $a_{\alpha} \subset \omega_{1}-\{\alpha\}$ such that $f_{\alpha} \in L_{\alpha}=\{f \in$ $\sigma(\mathscr{D}) \mid f(\alpha)=1$ and $f(\beta)=0$ for all $\left.\beta \in a_{\alpha}\right\} \subset L$. Since $B$ is uncountable, $B$ has an uncountable subset $B^{\prime}$ such that $\left\{a_{\alpha} \mid \alpha \in B^{\prime}\right\}$ forms a $\Delta$-system; that is, there is an $a \subset \omega_{1}$ such that $a_{\alpha} \cap a_{\beta}=a$ for all $\alpha$ and $\beta$ in $B^{\prime}$. Since $a$ is finite, there is $\gamma \in C-a$; but $f_{\gamma} \in F$ and $f_{\gamma}$ is a limit of $\bigcup_{\alpha \in B^{\prime}} L_{\alpha} \subset L$, as desired.

Proposition 2. If every finite subproduct of $\sigma$ is hereditarily normal and $\sigma$ is hereditarily $\kappa$-paracompact, then $\sigma$ is hereditarily normal.

Proof. Let $\mathscr{A}=\kappa^{<\omega}$. Let $G$ be an arbitrary open subset of $\sigma$ and $\mathscr{G}=$ $\left\{G_{1}, G_{2}\right\}$ a binary open cover of $G$. To prove that $\sigma$ is hereditarily normal, it 
suffices to find a closed cover $\left\{K_{1}, K_{2}\right\}$ of $G$ with $K_{i} \subset G_{i}$ for $i=1,2$. Let $U_{a, i}=\bigcup\left\{U \mid U\right.$ is open in $\left.Y_{a}, p_{a}^{-1}(U) \subset G_{i}\right\}$ for each $a \in \mathscr{A}$ and $i=1,2$, and put $U_{a}=\bigcup_{i=1}^{2} U_{a, i}$. Then $U_{a}$ is open in $Y_{a}$ and $\left\{p_{a}^{-1}\left(U_{a}\right) \mid a \in \mathscr{A}\right\}$ is an open cover of $G$ such that $p_{a}^{-1}\left(U_{a}\right) \subset p_{b}^{-1}\left(U_{b}\right)$ for each $a, b \in \mathscr{A}$ with $a \subset b$. Since $|\mathscr{A}|=\kappa$ and $G$ is $\kappa$-paracompact, there is a locally finite open cover $\mathscr{J}=\left\{J_{a} \mid a \in \mathscr{A}\right\}$ of $G$ such that $J_{a} \subset p_{a}^{-1}\left(U_{a}\right)$ for each $a \in \mathscr{A}$. Let $\Lambda=\mathscr{A}^{<\omega}$ and put $V_{\lambda}=G-\bigcup\left\{\operatorname{cl}\left(J_{b}\right) \mid b \in \mathscr{A}-\lambda\right\}$ for each $\lambda \in \Lambda$. Then

(1) $\mathscr{V}=\left\{V_{\lambda} \mid \lambda \in \Lambda\right\}$ is an open cover of $G$;

(2) if $\lambda, \mu \in \Lambda$ and $\lambda \subset \mu$, then $V_{\lambda} \subset V_{\mu}$; and

(3) let $a_{\lambda}=\bigcup\{a \mid a \in \lambda\}$ for each $\lambda \in \Lambda$; then $a_{\lambda} \in \mathscr{A}$ and $\operatorname{cl}_{G} V_{\lambda} \subset$ $p_{a_{\lambda}}^{-1}\left(U_{a_{\lambda}}\right)$.

Let $E_{\lambda}=\bigcup\left\{E \mid E\right.$ is open in $\left.Y_{a_{\lambda}}, p_{a_{\lambda}}^{-1}(E) \subset V_{\lambda}\right\}$ for each $\lambda \in \Lambda$. Then

(4) $P_{a_{\lambda}}^{-1}\left(E_{\lambda}\right) \subset V_{\lambda}$;

(5) $E_{\lambda} \subset U_{a_{\lambda}}$;

(6) if $\lambda \subset \mu$, then $p_{a_{\lambda}}^{-1}\left(E_{\lambda}\right) \subset p_{a_{\mu}}^{-1}\left(E_{\mu}\right)$; and

(7) $\left\{p_{a_{\lambda}}^{-1}\left(E_{\lambda}\right) \mid \lambda \in \Lambda\right\}$ is an open cover of $G$.

It is obvious that (4)-(6) hold.

Proof of (7). Let $x \in G$. Then $x \in V_{\lambda}$ for some $\lambda \in \Lambda$ by (1). Since $V_{\lambda}$ is open in $\sigma$, there are an $a \in \mathscr{A}$ and an open set $W$ in $Y_{a}$ such that $x \in$

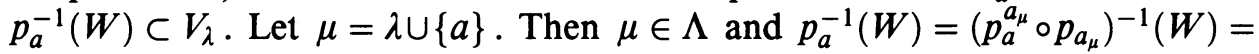
$p_{a_{\mu}}^{-1}\left(\left(p_{a}^{a_{\mu}}\right)^{-1}(W)\right)$. Since $\left(p_{a}^{a_{\mu}}\right)^{-1}(W)$ is open in $Y_{a_{\mu}},\left(p_{a}^{a_{\mu}}\right)^{-1}(W) \subset E_{\mu}$ by the definition of $E_{\mu}$. Thus $x \in p_{a_{\mu}}^{-1}\left(E_{\mu}\right)$.

Let $B_{\lambda}=\operatorname{cl} E_{\lambda} \cap\left(\operatorname{cl} U_{a_{\lambda}}-U_{a_{\lambda}}\right)$ for each $\lambda \in \Lambda$. Then

(8) $p_{a_{\lambda}}^{-1}\left(B_{\lambda}\right) \cap G=\varnothing$ for each $\lambda \in \Lambda$.

Proof of (8). Suppose there is an $x=\left(x_{\alpha}\right)_{\alpha<\kappa} \in p_{a_{\lambda}}^{-1}\left(B_{\lambda}\right) \cap G$. Then $p_{a_{\lambda}}(x) \in B_{\lambda}$. Thus $p_{a_{\lambda}}(x) \in \operatorname{cl} E_{\lambda}$. Since $E_{\lambda} \subset U_{a_{\lambda}}, p_{a_{\lambda}}(x) \in \operatorname{cl} U_{a_{\lambda}}$ and so $x \notin p_{a_{\lambda}}^{-1}\left(U_{a_{\lambda}}\right)$. Since $\operatorname{cl}_{G}\left(p_{a_{\lambda}}^{-1}\left(E_{\lambda}\right)\right) \subset \operatorname{cl}_{G} V_{\lambda} \subset p_{a_{\lambda}}^{-1}\left(U_{a_{\lambda}}\right), x \notin \operatorname{cl}_{G}\left(p_{a_{\lambda}}^{-1}\left(E_{\lambda}\right)\right)$. On the other hand, we have $x \in \mathrm{cl}_{G}\left(p_{a_{\lambda}}^{-1}\left(E_{\lambda}\right)\right)$. This is a contradiction. To show that $x \in \operatorname{cl}_{G}\left(p_{a_{\lambda}}^{-1}\left(E_{\lambda}\right)\right)$, let $H$ be an arbitrary open set such that $x \in H \subset G$. Then there are an $a \in \mathscr{A}$ and an open set $W_{a}$ in $Y_{a}$ such that $x \in p_{a}^{-1}\left(W_{a}\right) \subset H$. Let $\mu=\lambda \cup\{a\}$, and put $W_{\mu}=\left(p_{a}^{a_{\mu}}\right)^{-1}\left(W_{a}\right)$. Then $x \in p_{a_{\mu}}^{-1}\left(W_{\mu}\right) \subset H$ and $p_{a_{\lambda}}^{a_{\mu}}\left(W_{\mu}\right)$ is a neighborhood of $p_{a_{\lambda}}(x)$ in $Y_{a_{\lambda}}$ because $p_{a_{\lambda}}^{a_{\mu}}$ is an open map. Since $p_{a_{\lambda}}(x) \in \operatorname{cl} E_{\lambda}, E_{\lambda} \cap p_{a_{\lambda}}^{a_{\mu}}\left(W_{\mu}\right) \neq \varnothing$. Let $b \in E_{\lambda} \cap p_{a_{\lambda}}^{a_{\mu}}\left(W_{\mu}\right)$. Then $b=p_{a_{\lambda}}^{a_{\mu}}(c)$ for some $c \in W_{\mu}$. Let $y \in \sigma$ be an element such that $p_{a_{\mu}}(y)=c$. Then $p_{a_{\lambda}}(y)=b$. Therefore, $y \in p_{a_{\mu}}^{-1}\left(W_{\mu}\right) \cap p_{a_{\lambda}}^{-1}\left(E_{\lambda}\right)$. Thus $H \cap P_{a_{\lambda}}^{-1}\left(E_{\lambda}\right) \neq \varnothing$.

Since $U_{a_{\lambda}} \cap \mathrm{cl} E_{\lambda}=\left(\mathrm{cl} E_{\lambda}\right)-B_{\lambda} \subset \bigcup_{i=1}^{2} U_{a_{\lambda}, i},\left(\operatorname{cl} E_{\lambda}\right)-B_{\lambda}$ is closed in $Y_{a_{\lambda}}-B_{\lambda}$, and the $U_{a_{\lambda}, i}$ are open in $Y_{a_{\lambda}}$, there are closed sets $F_{a_{\lambda}, i}$ of $Y_{a_{\lambda}}-B_{\lambda}$ such that $F_{a_{\lambda}, i} \subset U_{a_{\lambda}, i}$ and $U_{a_{\lambda}} \cap \mathrm{cl} E_{\lambda}=\bigcup_{i=1}^{2} F_{a_{\lambda}, i}$. The $p_{a_{\lambda}}^{-1}\left(F_{a_{\lambda}, i}\right)$ are closed sets of $G$ because $p_{a_{\lambda}}^{-1}\left(F_{a_{\lambda}, i}\right)$ are closed sets in $p_{a_{\lambda}}^{-1}\left(Y_{a_{\lambda}}-B_{\lambda}\right)=\sigma-p_{a_{\lambda}}^{-1}\left(B_{\lambda}\right)$ and $p_{a_{\lambda}}^{-1}\left(F_{a_{\lambda}, i}\right) \subset G \subset \sigma-p_{a_{\lambda}}^{-1}\left(B_{\lambda}\right)$.

Since $\left\{p_{a_{\lambda}}^{-1}\left(E_{\lambda}\right) \mid \lambda \in \Lambda\right\}$ is an open cover of $G,|\Lambda|=\kappa$, and $G$ is $\kappa$ paracompact, there is a locally finite open cover $\left\{C_{\lambda} \mid \lambda \in \Lambda\right\}$ of $G$ such that 
$C_{\lambda} \subset p_{a_{\lambda}}^{-1}\left(E_{\lambda}\right)$ for each $\lambda \in \Lambda$. Let $K_{i}=\bigcup\left\{p_{a_{\lambda}}^{-1}\left(F_{a_{\lambda}, i}\right) \cap \operatorname{cl}_{G} C_{\lambda} \mid \lambda \in \Lambda\right\}$ for $i=1,2$. Then $K_{i}$ are closed sets in $G$ such that $K_{i} \subset G_{i}$ and $G=\bigcup_{i=1}^{2} K_{i}$.

Proof of Theorem 2. By Theorem $1, \sigma(\mathscr{D})$ is not hereditarily normal and thus, by Proposition $2, \sigma(\mathscr{D})$ is not hereditarily $\omega_{1}$-paracompact. Since every $\sigma$ for uncountable $\mathscr{S}$ contains a copy of $\sigma(\mathscr{D}), \sigma$ is not hereditarily $\omega_{1}$ paracompact.

Proposition 3. If every finite subproduct of $\sigma$ is normal and $\sigma$ is $\kappa$-paracompact, then $\sigma$ is normal.

Proof. Let $\mathscr{G}=\left\{G_{1}, G_{2}\right\}$ be an arbitrary binary open cover of $\sigma$. Let $U_{a, i}=$ $\bigcup\left\{U \mid U\right.$ is open in $\left.Y_{a}, p_{a}^{-1}(U) \subset G_{i}\right\}$ for each $a \in \mathscr{A}$ and $i=1,2$, and put $U_{a}=\bigcup_{i=1}^{2} U_{a, i}$. Let us define $\Lambda, \mathscr{J}$ and $\mathscr{V}$ as in the proof of Proposition 2. Let $T_{a_{\lambda}}=Y_{a_{\lambda}}-p_{a_{\lambda}}\left(\sigma-\mathrm{cl} V_{\lambda}\right)$ for each $\lambda \in \Lambda$. Then $T_{a_{\lambda}}$ is a closed subset of $Y_{a_{\lambda}}$ because $p_{a_{\lambda}}$ is an open map. Since $Y_{a_{\lambda}}$ is normal, there are open sets $M_{a_{\lambda}, i}$ in $Y_{a_{\lambda}}$ such that $T_{a_{\lambda}} \subset \bigcup_{i=1}^{2} M_{a_{\lambda}, i}$ and $\operatorname{cl} M_{a_{\lambda}, i} \subset U_{a_{\lambda}, i}$ for each $i=1,2$. Let $C_{\lambda}=$ int $P_{a_{\lambda}}^{-1}\left(T_{a_{\lambda}}\right)$. Then

(*) $\left\{C_{\lambda} \mid \lambda \in \Lambda\right\}$ is an open cover of $\sigma$.

Proof of $(*)$. This proof is quite similar to that of [1, Theorem 3.4]. For completeness we shall mention this. Let $x=\left(x_{\alpha}\right)_{\alpha<\kappa} \in \sigma$. Then $x \in V_{\lambda}$ for some $\lambda$. Since $V_{\lambda}$ is open in $\sigma$, there are an $a \in \mathscr{A}$ and an open set $W$ in $Y_{a}$ such that $x \in p_{a}^{-1}(W) \subset V_{\lambda}$. Let $\mu=\lambda \cup\{a\}$. Then $x \in C_{\mu}$ because $p_{a}^{-1}(W) \subset p_{a_{\mu}}^{-1}\left(T_{a_{\mu}}\right)$. To show that $p_{a}^{-1}(W) \subset p_{a_{\mu}}^{-1}\left(T_{a_{\mu}}\right)$, let $y \in p_{a}^{-1}(W)-p_{a_{\mu}}^{-1}\left(T_{a_{\mu}}\right)$. Then $p_{a}(y) \in W$ and $p_{a_{\mu}}(y) \notin T_{a_{\mu}}$ and so $p_{a_{\mu}}(y) \in p_{a_{\mu}}\left(\sigma-\operatorname{cl} V_{\lambda}\right)$. Thus $p_{a_{\mu}}(y)=p_{a_{\mu}}(z)$ for some $z \in \sigma-\operatorname{cl} V_{\lambda}$. Since $a \subset a_{\mu}, p_{a}(y)=p_{a}(z)$. Therefore, $p_{a}(z) \in W$ and so $z \in p_{a}^{-1}(W)$. Hence $p_{a_{\mu}}(z) \in T_{a_{\mu}}$. This is a contradiction. The proof of $(*)$ is complete.

Since $|\Lambda|=\kappa$ and $\sigma$ is $\kappa$-paracompact, there is a locally finite open cover $\left\{K_{\lambda} \mid \lambda \in \Lambda\right\}$ of $\sigma$ such that $K_{\lambda} \subset C_{\lambda}$ for each $\lambda \in \Lambda$. Let

$$
L_{i}=\bigcup_{\lambda \in \Lambda}\left(K_{\lambda} \cap p_{a_{\lambda}}^{-1}\left(M_{a_{\lambda}, i}\right)\right) \text { for } i=1,2 .
$$

Then $\sigma=\bigcup_{i=1}^{2} L_{i}$ and $\operatorname{cl} L_{i} \subset G_{i}$. Thus $\sigma$ is normal.

Proof of Theorem 3. The "if part" follows from (B) in $\S 1$. The "only if part" follows from Proposition 3.

Theorem 3 can be generalized as:

Theorem 5. Suppose every finite subproduct of $\sigma$ is $\kappa$-paracompact and normal. Then $\sigma$ is $\kappa$-paracompact iff $\sigma$ is normal.

Proposition 4. If every finite subproduct of $\sigma$ is hereditarily metacompact (resp. hereditarily countably metacompact), then $\sigma$ is hereditarily metacompact (resp. hereditarily countably metacompact).

Proof. For $n \in \omega$, let $\sigma_{n}=\left\{x \in X||\left\{\alpha \mid x(\alpha) \neq x^{*}(\alpha)\right\} \mid \leq n\right\} ; \sigma_{n}$ is closed in $\sigma$. Let $G$ be an arbitrary open subset of $\sigma$ and $\mathscr{G}$ an arbitrary (countable) open cover of $G$. For each $n<\omega$ and $a \in \kappa^{n}$, choose a point finite open cover $\mathscr{L}_{a}$ of $\left(Y_{a}-\sigma_{n-1}\right) \cap G$ which is a refinement of $\mathscr{G}$. For each $J \in \mathscr{J}_{a}$, 
choose $G_{J} \in \mathscr{G}$ such that $J \subset G_{J}$, and put $\mathscr{H}_{n}=\left\{p_{a}^{-1}(J) \cap G_{J}-\sigma_{n-1} \mid J \in \mathscr{J}_{a}\right.$, $\left.a \in \kappa^{n}\right\}$. Then $\mathscr{H}_{n}$ is a point finite open family in $G$. To show this, let $x \in G$ and suppose $x \in p_{a}^{-1}(J)-\sigma_{n-1}$ with $a \in \kappa^{n}$ and $J \in \mathscr{J}_{a}$. Then $p_{a}(x) \in J$ and so $p_{a}(x) \notin \sigma_{n-1}$. Thus $a \subset\left\{\alpha \in \kappa \mid x(\alpha) \neq x^{*}(\alpha)\right\}$.

It is obvious that $\mathscr{H}_{n}$ is a cover of $\left(\sigma_{n}-\sigma_{n-1}\right) \cap G$. Let $\mathscr{H}=\bigcup_{n<\omega} \mathscr{H}_{n}$. For each $x \in G, x \in \sigma_{n}-\sigma_{n-1}$ for some $n$. Thus $x \notin H$ for each $H \in \bigcup_{m \geq n} \mathscr{H}_{m}$. Therefore, $\mathscr{H}$ is a point finite open cover of $G$ which is a refinement of $\mathscr{G}$.

Proof of Theorem 4. The "if part" follows from Proposition 4 because countable paracompactness coincides with countable metacompactness for normal spaces. The "only if part" follows from Proposition 2.

\section{ACKNOWLEDGMENT}

The author would like to thank the referee for kindly providing extensive modifications to improve this paper's readability. The proof of Theorem 1 was simplified by the referee.

\section{REFERENCES}

1. A. Bešlagić, Normality in products, Topology Appl. 22 (1986), 71-82.

2. D. K. Burke, Covering properties, Handbook of Set Theoretic Topology, Chapter 9 (K. Kunen and J. Vaughan, ed.), North-Holland, Amsterdam, 1984, pp. 347-422.

3. H. H. Corson, Normality in subsets of product spaces, Amer. J. Math. 81 (1959), 785-796.

4. A. P. Kombarov, On the normality of $\Sigma_{m}$-products, Soviet Math. Dokl. 14 (1973), 10501053.

5. K. Morita, Paracompactness and product spaces, Fund. Math. 50 (1962), 223-236.

Department of Mathematics, Faculty of Science, Shizuoka University, Ohya, SHIZUOKA, 422 JAPAN 